\title{
Observations of multiple populations in star clusters
}

\author{
Giampaolo Piotto \\ Dipartimento di Astronomia, Università di Padova, \\ Vicolo dell'Osservatorio, 3, I-35122, Padova, Italy \\ email: giampaolo.piotto@unipd.it
}

\begin{abstract}
An increasing number of photometric observations of multiple stellar populations in Galactic globular clusters is seriously challenging the paradigm of GCs hosting single, simple stellar populations. These multiple populations manifest themselves in a split of different evolutionary sequences as observed in the cluster color-magnitude diagrams. Multiple stellar populations have been identified in Galactic and Magellanic Cloud clusters. In this paper we will summarize the observational scenario.
\end{abstract}

Keywords. globular clusters, stars: horizontal-branch, stellar populations, techniques: photometric, astrometry

\section{Introduction}

Globular star clusters (GC) have occupied a prominent role in our understanding of the structure and evolution of (low mass) stars. At the basis of the use of GCs as templates for stellar models was the assumption that their stars can be idealized as "simple stellar populations" (SSP), i.e. as an assembly of coeval, initially chemically homogeneous, single stars. Thanks to this idea, GCs, and star clusters in general, have been used for decades to test and calibrate synthetic models of stellar populations, a critical tool for studying galaxies at low, as well as at high, redshift.

Color-magnitude diagrams (CMD), like the magnificent CMD of NGC 6397 by Richer et al. (2008, see also Anderson et al. 2008), fully support the paradigma of GCs hosting simple stellar populations. However, there is a growing body of observational facts which challenge this traditional view. Since the eighties, we have known that GCs show a peculiar pattern in their chemical abundances (see Gratton et al. 2004 for a recent review). While they are generally homogeneous insofar Fe-peak elements are considered, they often exhibit large anticorrelations between the abundances of $\mathrm{C}$ and $\mathrm{N}, \mathrm{Na}$ and $\mathrm{O}$, $\mathrm{Mg}$ and $\mathrm{Al}$. These anticorrelations are attributed to the presence at the stellar surfaces of a fraction of the GC stars of material which have undergone $\mathrm{H}$ burning at temperatures of a few ten millions $\mathrm{K}$ (Prantzos et al. 2007; less for the $\mathrm{C}$ and $\mathrm{N}$ anticorrelation). This pattern is peculiar to GC stars; field stars show only changes in $\mathrm{C}$ and $\mathrm{N}$ abundances, expected from the typical evolution of low-mass stars (Sweigart \& Mengel 1979); this pattern in GC stars is primordial, since it is observed in stars at all evolutionary phases (Gratton et al. 2001); and the whole star is involved (Cohen et al. 2002).

In addition, since the sixties (Sandage and Wildey 1967, van den Bergh 1967), we know that the horizontal branches (HB) of some GCs can be rather peculiar. This problem, usually known as the the second parameter problem, still lacks a comprehensive understanding: many mechanisms, and many parameters have been proposed to explain the HB peculiarities, but none apparently is able to explain the entire observational scenario. It is quite possible that a combination of parameters is responsible for the HB morphology 


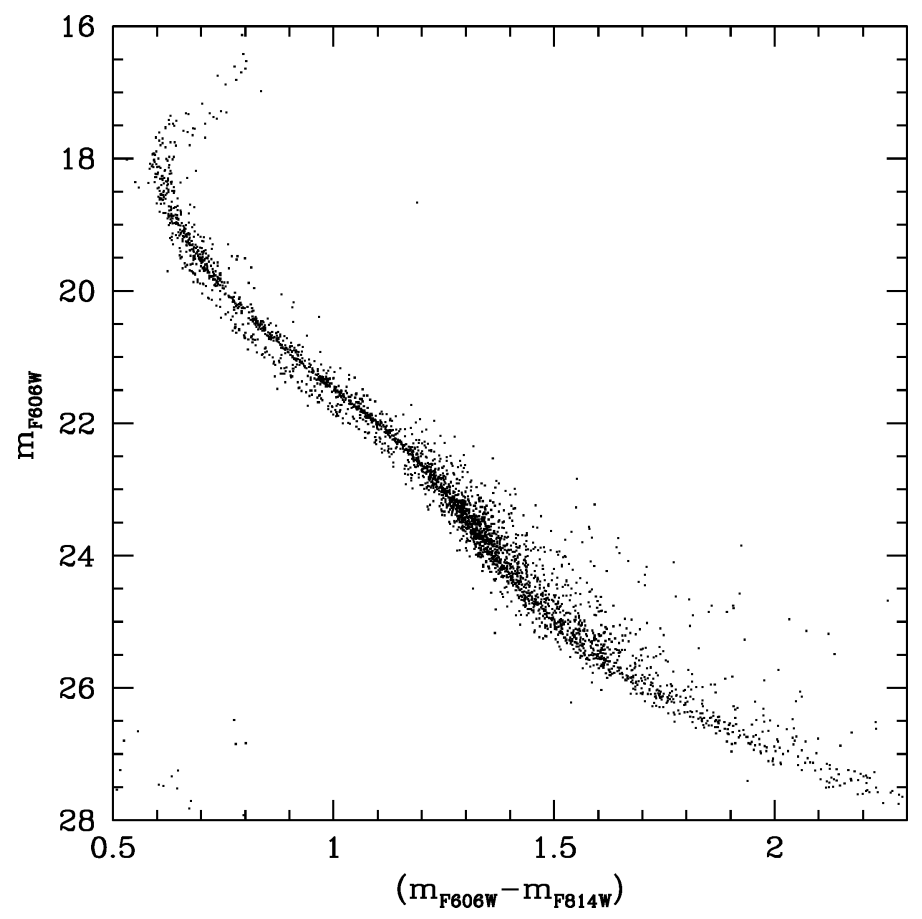

Figure 1. The multiple MS of Omega Centauri. This spectacular CMD (from Bedin et al. 2009, in preparation) comes from multi-epoch observations of a field at 17 arcmin from the cluster center. The plotted stars have been selected on the basis of their proper motion and all of them are cluster members. The split of the two MSs is clearly visible from the TO down to $m_{F 606 W}=23.0$. Then the two MS seem to merge, due to the increased photometric error. Some stars are on the red side of the two main MSs; they are too far from the red MS to be binaries. These stars correspond to the third MS discussed in Villanova et al. (2007), which is likely related to the RGB-a of Pancino et al. (2002).

(Fusi Pecci et al. 1993). Surely, the total cluster mass seems to be a relevant parameter (Recio-Blanco et al. 2006).

It is tempting to relate the second parameter problem to the complex abundance pattern of GCs. Since high $\mathrm{Na}$ and low $\mathrm{O}$ abundances are signatures of material processed through hot H-burning, they should be accompanied by high He-contents (D'Antona \& Caloi 2004). In most cases, small He excesses up to $\delta Y \sim 0.04$ (that is $\mathrm{Y} \sim 0.28$, assuming the original He content was the Big Bang one) are expected. While this should have small impact on the colors and magnitudes of stars up to the tip of the RGB, a large impact is expected on the colors of the HB stars, since He-rich stars should be less massive. For example, in the case of GCs of intermediate metallicity ( $[\mathrm{Fe} / \mathrm{H}] \sim-1.5)$, the progeny of He-rich, Na-rich, O-poor RGB stars should reside on the blue part of the HB, while those of the "normal" He-poor, Na-poor, O-rich stars should be within the instability strip, or redder than it. Actually, mean $\mathrm{HB}$ colors are influenced by the mass loss along the RGB and by small age differences of 2-3 Gyr. However, within a single GC a correlation is expected between the distribution of masses (i.e. colors) of the HB-stars and of $\mathrm{Na}$ and O abundances.

In summary, a number of apparently independent observational facts seems to suggest that, at least in some GCs, there are stars which have formed from material which must have been processed by a previous generation of stars. 

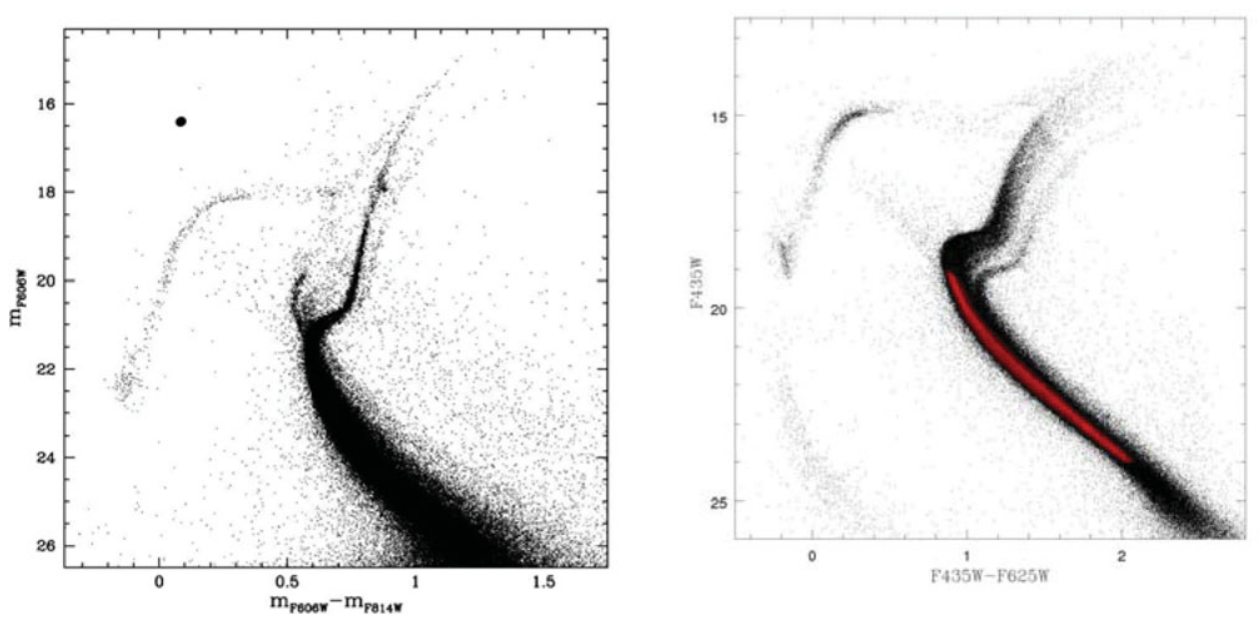

Figure 2. The CMD of M54 (left panel) resembles the CMD of $\omega$ Cen in many aspects. Do the two clusters have a similar origin?

The questions is: do we have some direct, observational evidence of the presence of multiple populations in GCs? Very recent discoveries, made possible by high accuracy photometry on deep HST images, allowed us to positively answer to this question. In this paper, we will summarize these new observational facts, and briefly discuss their link to the complex abundance pattern and to the anomalous HBs.

\section{Observational evidence of multiple populations in GCs}

\subsection{The first discoveries}

The first, direct observational evidence of the presence of more than one stellar population in a GC was published by Bedin et al. (2004). Bedin et al. found that, for a few magnitudes below the turn-off (TO), the main sequence (MS) of $\omega$ Centauri splits in two (Fig. 1). Indeed, the suspicion of a MS split in $\omega$ Cen was already raised by Jay Anderson in his $\mathrm{PhD}$ thesis, but the result was based on only one external WFPC2 field, and this finding was so unexpected that he decided to wait for more data and more accurate photometry to be sure of its reality. Bedin et al. (2004) confirmed the MS split in Anderson's field and in an additional ACS field located 17 arcmin from the cluster center. Now, we know that the multiple MS is present throughout the cluster, although the ratio of blue to red MS stars diminishes going from the cluster core to its envelope (Sollima et al. 2007, Bellini et al. 2009, in preparation). We also know that there is a third, redder MS (see Fig. 1), containing about $5 \%$ of the $\omega$ Cen MS stars (Villanova et al. 2007), probably related to the most metal rich RGB-a of Pancino et al. (2002).

The more shocking discovery concerning the multiple populations in $\omega$ Cen, however, came from a follow-up spectroscopic analysis that showed that the blue MS has twice the metal abundance of the dominant red branch of the MS (Piotto et al. 2005). The only isochrones that would fit this combination of color and metallicity were extremely enriched in helium $(Y \sim 0.38)$ relative to the dominant old-population component, which presumably has primordial helium.

Indeed, the scenario in $\omega$ Cen is even more complex. As is already evident in the CMD of Bedin et al. (2004), the three MSs of $\omega$ Cen spread into a highly multiple sub-giant 
branch (SGB), with five distinct components, characterized by different metallicities and ages (Sollima et al. 2005, Villanova et al. 2007; the latter has a detailed discussion.)

These results reinforced the suspicion that the multiple MS of $\omega$ Cen could just be an additional peculiarity of an already anomalous object, which might not even be a GC, but a remnant of a dwarf galaxy instead.

On this respect, it might be instructive to compare the CMD of $\omega$ Cen (Fig 2, right panel), with the CMD of M54 (Fig. 2, left panel). The two CMDs look rather similar. We know that M54 almost coincides with the nucleus of the disrupting Sagittarius dwarf galaxy. And the complexity of the CMD of M54 of Fig. 2 is indeed due to the fact that we observe, in the same field, both M54 stars and background/foreground stars of the Sagittarius dwarf nucleus. M54 might have originated in the nucleus of its hosting galaxy, or ended there from elsewhere as a consequence of dynamical friction (Bellazzini et al. 2008). The important fact here is that both M54 and the Sagittarius nucleus now are located in the same place, in mutual dynamical interaction. It is very tempting to think that, a few Gyrs ago, $\omega$ Cen could have been exactly what we now find in the nucleus of the Sagittarius.

The spectacular case of $\omega$ Cen stimulated a number of investigations which showed that the multiple-population scenario is not a peculiarity of a single object. Piotto et al. (2007) showed that also the CMD of NGC 2808 is split into three MSs. Because of the negligible dispersion in Fe peak elements (Carretta et al. 2006), Piotto et al. (2007) proposed the presence of three groups of stars in NGC 2808, with three different He contents, in order to explain the triple MS. These groups may be associated to the three groups with different Oxygen content discovered by Carretta et al. (2006). These results are also consistent with the presence of a multiple, extended HB, as discussed thoroughly in D'Antona and Coloi (2004) and D'Antona et al. (2006).

Also NGC 1851 must have at least two, distinct stellar populations. In this case the observational evidence comes from the split of the SGB in the CMD of this cluster obtained from ACS/HST data (Milone et al. 2008a). More recently, Peter Stetson (see paper in the present Proceedings) has identified the SGB split also in the cluster envelope, thanks to spectacular photometry from ground-based wide field images. If the magnitude difference between the two SGBs were due only to an age difference, the two star-formation episodes would have to have been separated by at least 1 Gyr. However, as shown by Cassisi et al. (2007), the presence in NGC 1851 of two stellar populations, one with a normal $\alpha$-enhanced chemical composition, and one characterized by a strong CNO-Na anticorrelation pattern, could reproduce the observed CMD split. In this case, the age spread between the two populations could be much smaller, possibly consistent with the small age spread implied by the narrow TO of NGC 2808. In other words, the SGB split would be mainly a consequence of the metallicity difference, and only negligibly affected by (a small) age dispersion. The Cassisi et al. (2007) hypothesis is supported by the presence of a group of CN-strong and a group of CN-weak stars discovered by Hesser et al. (1982), and by a recent study by Yong \& Grundahl (2008) who find a Na-O anticorrelation among NGC 1851 giants.

NGC 1851 is considered a sort of prototype of bimodal-HB clusters. Milone et al. (2008a) note that the fraction of fainter/brighter SGB stars is remarkably similar to the fraction of bluer/redder HB stars. Therefore, it is tempting to associate the brighter SGB stars to the CN-normal, s-process element-normal stars and to the red HB, while the fainter SGB should be populated by CN-strong, s-process element-enhanced stars which should evolve into the blue HB. In this scenario, the faint SGB stars should be slightly younger (by a few $10^{7}$ to a few $10^{8}$ years) and should come from processed material 


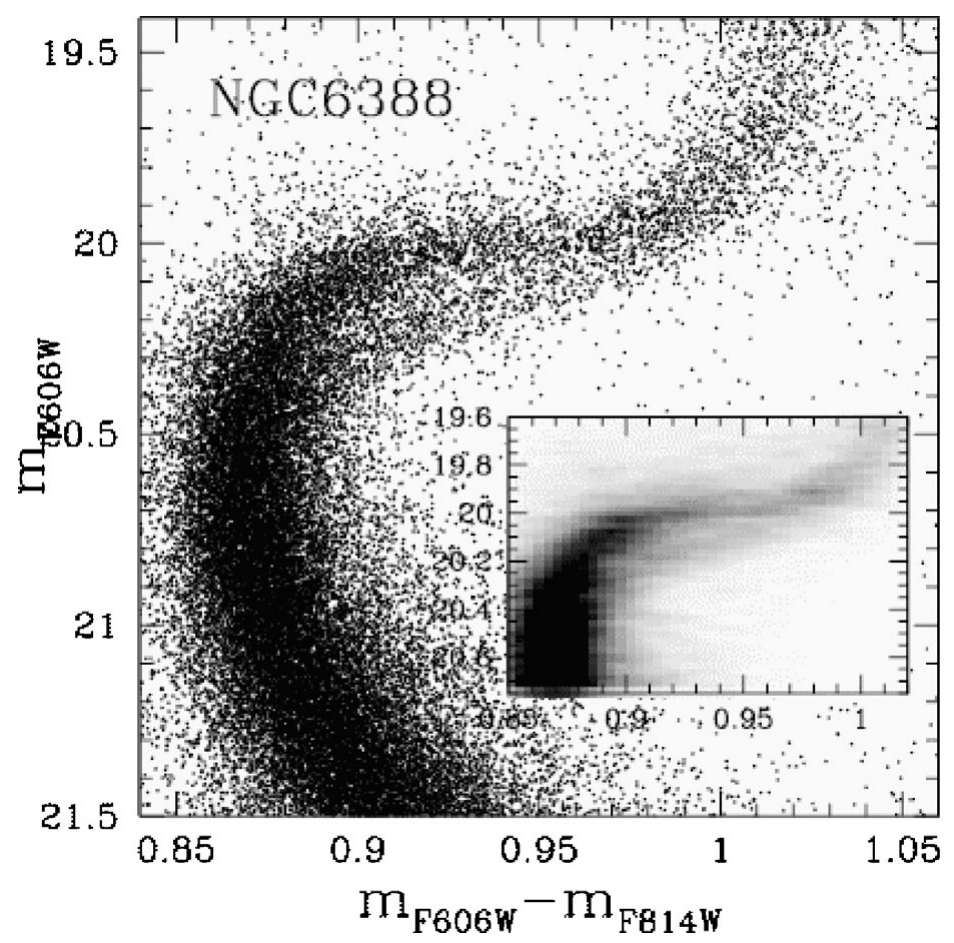

Figure 3. The double SGB in NGC 6388.

which might also be moderately He enriched, a fact that would help explain why they evolve into the blue HB. By studying the cluster MS, Milone et al. (2008a) exclude an He enrichment larger than $\Delta Y=0.03$, as expected also by the models of Cassisi et al. (2007). Nevertheless, this small He enrichment, coupled with an enhanced mass loss, would be sufficent to move stars from the red to the blue side of the RR Lyrae instability strip. Direct spectroscopic measurements of SGB and HB stars in NGC 1851 are badly needed.

\subsection{More recent findings. I. Galactic GCs}

Prompted by the results on $\omega$ Cen, NGC 2808, and NGC 1851 we used HST archive images and new proprietary data (GO10922 and GO11233, PI Piotto) to search for multiple populations. We are still working on the optimization of the software for the optimal extraction of high-accuracy photometry from the WFPC2 images (in particular for the new data acquired in the last months). For the moment, we did not find another cluster with multiple MSs as in $\omega$ Cen and NGC 2808, thought there are a couple of suspected cases.

However, we did find many clusters (at least seven, at the moment) with a double SGB (Piotto et al. 2009, in preparation). Among these, the most interesting cases are those of NGC 6388 (Fig. 3), M22 (Fig. 4), and M54 (Fig. 5).

Figure 3 shows that, even after correction for differential reddening, the SGB of NGC 6388 closely resembles the SGB of NGC 1851. The results, originally coming from HST data, have been recently confirmed also using near-IR, multi-conjugate adaptive optics images (Moretti et al. 2008) collected with MAD@VLT. NGC 6388, as well as its twin cluster NGC 6441, are two extremely peculiar clusters. Since Rich et al. (1997), we know 


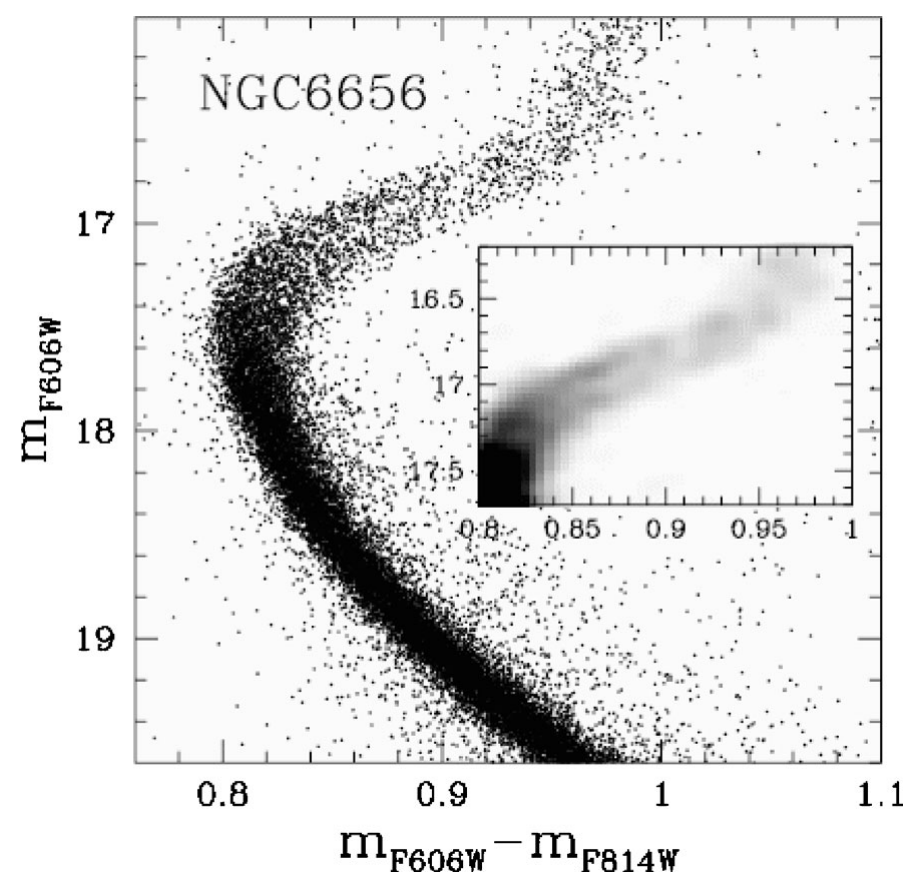

Figure 4. The double SGB in NGC 6656 (M22).

that, despite their high metal content - higher than in 47 Tucanae - they have a bimodal HB, which extends to extremely hot temperatures (Busso et al. 2007), totally un-expected for this metal rich cluster. NGC 6388 stars also display a Na-O anticorrelation (Carretta et al. 2007). Unfortunately, the available data do not allow us to study the MS of this cluster, to search for a MS split. In this context, it is worth noting that Caloi and D'Antona (2007), in order to reproduce the HB of NGC 6441, propose the presence of three populations, with three different He contents, one with an extreme He enhancement of $\mathrm{Y}=0.40$. Such a strong enhancement should be visible in a MS split, as in the case of $\omega$ Cen and NGC 2808. A strong He enhancement and a consequent MS split may also apply to NGC 6388, because of the many similarities with NGC 6441. As soon as the new WF3 and the restored ACS instruments at HST will be available, we plan to test these predictions (GO11739, PI Piotto).

The case of M22 (Fig. 4) is a very interesting one. For decades this cluster has been suspected to have metallicity variations, including a spread in $[\mathrm{Fe} / \mathrm{H}]$. The iron spread has been controversial until very recently, when, thanks to the aquisition of UVES@VLT high-resolution spectra of RGB stars, we (Marino et al. 2009, in preparation) could demonstrate that, not only the $[\mathrm{Fe} / \mathrm{H}]$ spread is confirmed, but that, indeed, there is a bimodal distribution in the iron content, and that this distribution is correlated with the abundance of s-process elements (as Y, Zr, Ba): Stars rich in Fe and Ca are also s-process element rich. The bimodal distribution of the SGB stars in M22 shown in Fig. 4 might be related to these two metallicity groups.

Also M54 shows a double SGB (Fig. 5). The fact that the stars populating the two SGBs are members of the M54 GC, and not field stars, is confirmed by the fact that they share exactly the same radial distribution within our ACS images centered on the cluster center. 


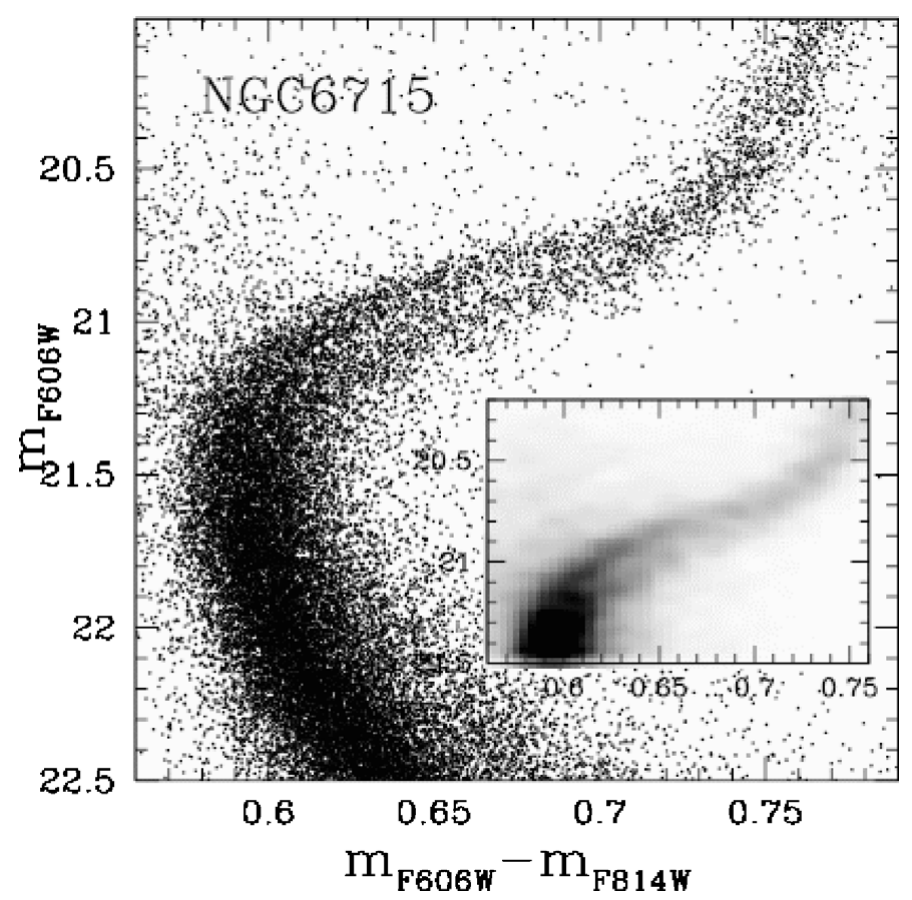

Figure 5. The double SGB in NGC 6715 (M54).

Piotto et al. (2009) shows that the double SGB phenomenon is quite common among massive GCs, though the fraction of stars in the two SGBs varies from cluster to cluster.

\subsection{More recent findings. II. Magellanic Cloud clusters}

The multiple-population phenomenon in star clusters is not confined to Galactic GCs only. The suspicion that some clusters in the Large Magellanic Cloud (LMC) could host more than one generation of stars has been raised in the past (e.g., Vallenari et al. 1994, Bertelli et al. 2003). However, only when high-precision photometry from ACS/HST images became available, could Mackey \& Broby Nielsen (2007) clearly demonstrate the presence of two populations, with an age difference of $\sim 300 \mathrm{Myr}$, in the 2 Gyr old cluster NGC 1846, in the LMC. In this case, the presence of the two populations is inferred by the presence of two TOs in the CMD. Mackey et al. (2008) identified two additional LMC clusters with multiple populations. More recently, Milone et al. (2008b), from the analysis of the CMDs of 16 intermediate age LMC clusters using HST archive data, showed that the multiple population phenomenon might be rather common among LMC clusters: $11(70 \%$ !) have CMDs which are not consistent with the presence of a single, simple stellar population (see also Kozhurina-Platais et al. poster at this meeting). Also the Small Magellanic Cloud seems to host a cluster with a CMD that is not consistent with a single stellar population (Glatt et al. 2008).

\section{An alternative approach to the search for multiple populations}

The presence of abundance spreads in GC stars is well known. As already mentioned, in some cases more metallicity groups of stars can be isolated in the same cluster, as in the case of NGC 2808 (Carretta et al. 2006). 
However, there is a recent finding for the GC M4 which is worth describing in some detail. Using high-resolution UVES@VLT spectra of more than 100 giants, Marino et al. (2008) have shown that also in this cluster, stars show a well defined Na-O anticorrelation (as in all GCs studied so far, searching for this phenomenon). The important result is that the distribution in $\mathrm{Na}$ (or O) content is clearly bimodal (see inset of Fig. 6), and this bimodal distribution is correlated with a bimodal distribution in CN strength among M4 stars. Stars that are Na-rich are also CN-strong.

The bimodality is also visible in the CMDs built using U-band images, as shown in Fig. 6. This is due to the strong effect of the $\mathrm{CN}$ bands on the $\mathrm{U}$ magnitude, as demonstrated by Marino et al. (2008). This is an interesting feature. Also in the case of M22, where Marino et al. (2009) have identified two groups of stars with different metal contents (see above), the $\mathrm{U}$ vs (U-V) CMD allows us to distinguish the two different populations. This RGB split or broadening in the U vs (U-B) CMD may be an alternative way to search for multiple stellar populations in GCs, in particular for clusters where large samples of high-resolution spectra are not available, or are not easily obtainable.

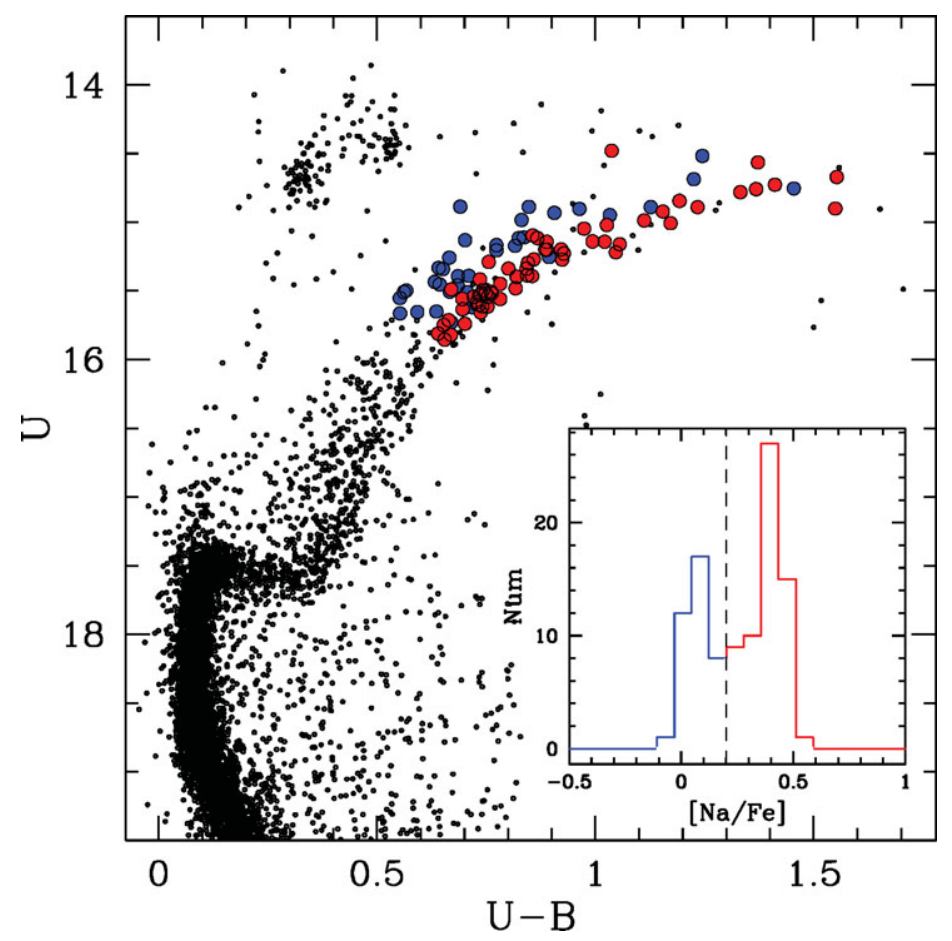

Figure 6. The $\mathrm{Na}$ and $\mathrm{O}$ distributions in M4 are bimodal (see inset). The bimodal distribution in these chemical elements is reflected also in a bimodal distribution of stars along the RGB.

\section{Discussion}

In the previous sections we have summarized the direct evidence we have of multiple generations of stars in star clusters. The observational scenario is rather complex. So far, multiple populations have been indentified by the presence of:

- multiple, distinct MSs, as in the case of $\omega$ Cen and NGC 2808;

- TO-SGB splits, in seven Galactic GCs, eleven intermediate-age LMC clusters, and at least one SMC cluster; 
- bimodal or multimodal distributions of light elements, such as $\mathrm{Na}$ or $\mathrm{O}$, or in the CN-strength, in some case associated with a broadening or multimodal distribution of RGB stars in the CMD involving the U-band.

In general, the multiple-population phenomenon differs from cluster to cluster, in the way it shows itself, in the ratio of the different populations present in the same cluster, and in the separation of the different sequences. An important property, shared by all the clusters where the phenomenon has been seen so far, is that the different populations are distinct. Only in some LMC clusters does there seem to be a broadened distribution, but it is not clear whether this is just due to photometric errors, which do not allow the separation of the single sequences, or is an intrinsic feature (Milone et al. 2008b).

At the moment, we cannot say whether the three different manifestations of the multimodality of cluster stellar populations reflect a single phenomenon. For example, it has been proposed (Bekki \& Mackey 2008) that the origin of the bimodal populations in LMC clusters could come from an encounter of a young cluster with a giant molecular cloud, where the formation of a second generation of stars is triggered by the encounter itself. On the other hand, the multiple populations identified in the (generally more massive) Galactic globular clusters could be due to a second (or third) generation of stars which formed from material polluted by the ejecta from a variety of possible firstgeneration stars (see review by $\mathrm{Yi}$ in these proceedings). For sure, these GCs are clearly not simple, single-stellar-population objects. The emerging evidence is that the starformation history can vary strongly from cluster to cluster, and that some GCs are able to produce very unusual objects, as no such He-rich MS stars have ever been found elsewhere. Reconstruction of this star-formation history requires a better understanding of the chemical-enrichment mechanisms, since not only the site of hot H-burning proposed to explain the He enhancement, but also the $\mathrm{NaO}$ anticorrelation, remains unclear. There are two requirements: (i) the temperature should be high enough; and (ii) the stars where the burning occur should be able to give back the processed material to the intracluster matter at a velocity low enough that it can be kept within the GC itself (a few tens of $\mathrm{km} / \mathrm{s}$ ). Candidates include: (i) Massive $\left(M>10 M_{\odot}\right)$ rotating stars (Decressin et al. 2007); (ii) the most massive among the intermediate-mass stars undergoing hot-bottom burning during their AGB phase (Ventura et al. 2001), and possibly (iii) Population III stars (again see Yi's contribution in these proceedings). The first two mechanisms act on different timescales $\left(10^{7}\right.$ and $10^{8} \mathrm{yr}$, respectively), and each solution has its pros and cons (Renzini 2008). The massive-star scenario should avoid mixing the O-poor, Na-rich material with that material that is rich in heavy elements from SNe, while it is not clear how the chemically processed material could be retained by the proto-cluster in spite of the fast winds and SN explosions always associated with massive stars. Producing the right pattern of abundances from massive AGB stars seems to require considerable fine tuning. In addition, both scenarios require that either the IMF of GCs was very heavily weighted toward massive stars, or that some GCs should have lost a major fraction of their original population (Bekki \& Norris 2006), and even then may be the remnants of tidally disrupted dwarf galaxies, as suggested by the complexity in the CMDs of $\omega$ Cen and of M54.

The observational scenario is becoming more complex, but, new results might have indicated the right track for a comprehensive understanding of the formation and early evolution of GCs. We are perhaps, for the first time, close to solving what has been for decades, and still, is a broken puzzle.

Acknowledgements. I wish to warmly thank Jay Anderson, Andrea Bellini, Luigi R. Bedin, Santi Cassisi, Ivan King, Antonino P. Milone, Alessia Moretti, and Sandro 
Villanova, without whom most of the results presented in this review would not have been possible. A special thanks to Alvio Renzini and Raffaele Gratton for many enthusiastic discussions on the subject of multi-populations in star clusters. I acknowledge partial support by MIUR (PRIN2007) and ASI under contract ASI-INAF I/016/07/0.

\section{References}

Anderson, J. et al. 2008, AJ, 135, 2114

Bedin, L. R., Piotto, G., Anderson, J., Cassisi, S., King, I. R., Momany, Y., \& Carraro, G. 2004, ApJ, 605, L125

Bekki, K. \& Mackey, A. D. 2008, arXiv/0812.0631

Bekki, K. \& Norris, J. E. 2006, ApJ (Letters), 637, L109

Bellazzini, M. et al. 2008, AJ, 136, 1147

Busso, G. et al. 2007, A\&\&A, 474, 105

Caloi, V. \& D'Antona, F. 2007, A\&A, 463, 949

Carretta, E., Bragaglia, A., Gratton, R. G., Leone, F., Recio-Blanco, A., \& Lucatello, S. 2006, $A \mathscr{G} A, 450,523$

Carretta, E. et al. 2007, A\&SA, 464, 957

Cassisi, S., Salaris, M., Pietrinferni, A., Piotto, G., Milone, A. P., Bedin, L. R., \& Anderson, J. 2007, ApJ, 672, L115

Cohen, J. G., Briley, M. M., \& Stetson, P. B. 2002, AJ, 123, 2525

D'Antona, F. \& Caloi, V. 2004, ApJ, 611, 871

D'Antona, F., Bellazzini, M., Caloi, V., Pecci, F. Fusi, Galleti, S., \& Rood, R. T. 2006, ApJ, 631,868

Decressin, T., Meynet, G., Charbonnel, C., Prantzos, N., \& Ekstrm, S. 2007, A\&A, 464, 1029

Glatt et al. 2008, AJ, 136, 1703

Gratton, R. et al. 2001, A\&A A, 369, 87

Gratton, R., Sneden, C., \& Carretta, E. 2004, ARAA, 42, 385

Hesser, J. E., Bell, R. A., Harris, G. L. H., \& Cannon, R. D. 1982, AJ, 87, 1470

Mackey, A. D. \& Broby Nielsen, P. 2007, MNRAS, 379, 151

Milone, A. P. et al. 2008a, ApJ, 673, 241

Milone, A. P. et al. 2008b, in press, arXiv0810.2558

Moretti, A. et al. 2008, in press, arXiv0810.2248

Piotto, G., et al. 2005, ApJ, 621, 777 (P05)

Piotto, G., et al. 2007, ApJ, 661, L53 (P07)

Prantzos, N., Charbonnel, C., \& Iliadis, C. 2007, A\& $A$, 470, 179

Recio-Blanco, A., Aparicio, A., Piotto, G., de Angeli, F., \& Djorgovski, S. G. 2006, A $ళ A, 452$, 875

Renzini, A. 2008, MNRAS, 391, 354

Richer, H. et al. 2008, AJ, 135, 2141

Sandage, A. \& Wildey, R. 1967, ApJ, 150, 469

Siegel et al. 2007, ApJ, 667, L57

Sollima, A., Pancino, E., Ferraro, F. R., Bellazzini, M., Straniero, O., \& Pasquini, L. 2005, ApJ, 634,332

Sollima, A., Ferraro, F. R., Bellazzini, M., Origlia, L., Straniero, O., \& Pancino, E. 2007, ApJ, 654,915

Sweigart, A. V. \& Mengel, J. G. 1979, ApJ, 229, 624

Ventura, P., D’Antona, F., Mazzitelli, I., \& Gratton, R. 2001, ApJ (Letters), 550, L65

Vallenari, A., Aparicio, A., Fagotto, F., Chiosi, C., Ortolani, S., \& Meylan, G. 1994, A\&A, 284, 447

Villanova, S., et al. 2007, ApJ, 663, 296

van den Bergh, S. 1967, $A J, 72,70$

Yong, D. \& Grundahl, F. 2008, ApJ, 672, L29 


\section{Discussion}

G. Meynet: 1) I would expect that He-rich stars are also those showing the greatest Na enrichment. Therefore stars on the blue main sequence of, e.g., NGC 2808, should show also the greatest $\mathrm{Na}$ enrichment. Is this the case? Is there any observational evidence of that? 2) The models which are used to derive the $\mathrm{Na}$ and $\mathrm{O}$ abundances, do they account for the fact that the star may be He- rich? If it is not the case, would this fact, if taken into account, change somewhat the results? Might it produce a less continuous Na- ratio distribution, reflecting the separated sequences derived in the CMD?

G. Piotro: 1) There is no direct measure of Na or O in MS stars in NGC 2808. However, I note that, accounting for the evolutionary times, the fraction of stars in the three MSs is such that we can link the reddest MS with the O-normal stars and the red sequence of the $\mathrm{HB}$, the intermediate MS with the O-poor stars and the EBT1 part of the HB as defined by Bedin et al. (2000, A\&A, 363, 159), and finally the bluest MS with the O-poorest group of stars and with the EBT2 HB. The last, hottest HB could be associated with the binary population; see Piotto et al. (2007, ApJ, 661, L53) for a more detailed discussion. 2) The models of Francesca DAntona and collaborators do take into account the He enhancement in the Na-O relation and in this way they can account for both the HB morphologies and the MS split observed, e.g., in NGC 2808 (see, e.g., DAntona et al. 2005, ApJ, 631, 868). If your question refers to the fact that strong He enhancement affects the precise models used to measure the abundances of the stars in the He-enhanced main sequences, I can answer that we did this check in Piotto et al. (2005 ApJ, 621, 777). We concluded that our He enhancement, up to $Y=0.4$, is affecting the measured metallicity of the blue main sequence of $\omega$ Cen by 0.02-0.03 dex, well within our measurement error.

A. DotTer: You drew a correlation between M54 and $\omega$ Cen, but while $\omega$ Cen shows a small age dispersion and large He dispersion, M54 has a large age dispersion and no evidence for enhanced He.

G. Piotro: I do not think we can say that M54 has no evidence of He enhancement: simply it has not yet been properly observed to infer the presence of He enhancement. As for the second point, it is true that M54 shows a less-dispersed SGB than $\omega$ Cen, but what this means in terms of age dispersion remains to be established, for both clusters. However, if you take the M54 and the Sagitarius nucleus together, they do show a large dispersion along the SGB. And now, try to imagine how the CMD of the M54 field will look a few Gyr from now.

V. Poole: Have you noticed any trends about when multiple populations occur in globular clusters?

G. Piotro: No. However, note that this is a new field, and we know too few clusters with clear evidence of multiple populations. It is still too early to identify any trend. Also, note that the multiple population evidence is different in different clusters.

C. Deliyannis: You showed a population of dwarfs in $\omega$ Cen that have $40 \%$ helium. Do you have any suggestions as to how $\omega$ Cen can create such a population?

G. Piotro: I am as concerned as you are about the possible presence of a stellar population with $Y=0.40$. Unfortunately, at the moment this solution seems to be the only 
one that can account for both the photometric and spectroscopic observations. As for the origin of such a high He abundance, see the review by $\mathrm{Yi}$ in these proceedings.

A. Sarajedini: A comment about the association of a $\omega$ Cen with M54, as nuclei of disrupted dwarfs: Bellazzini et al. (2008, arxiv:0807.0105) show that M54 is not likely to be the nucleus of Sgr. It moved to its current location from somewhere else.

G. Piotro: I am perfectly aware of the conclusions by Bellezini et al. (2008). My point in my talk is independent of whether or not M54 was born at the center of the Sagittarius dwarf galaxy or that it ended there because of dynamical friction. The fact is that, today, M54 and the nucleus of the Sgr dwarf galaxy are at the same place, and, therefore, they will dynamically evolve (mix) together. In a few billion years, M54 and the Sgr nucleus CMD will look very, very similar to the present day CMD of $\omega$ Cen, and, eventually the surrounding Sgr dwarf galaxy will have been completely stripped away by the Galactic tidal field.

H. Richer: It would be nice to measure the proposed high He abundance in $\omega$ Cen directly. Have you tried to see it among hot stars on the horizontal branch?

G. Piotro: Direct measurement of He in HB stars can be performed only in a narrow temperature interval: stars hotter than the Grundahl jump $(\sim 11,000 \mathrm{~K})$ are affected by He sedimentation and metal levitation; stars cooler than $\sim 9,000 \mathrm{~K}$ do not show He lines. We have recently proven the feasibility of this measurement for NGC 6752 stars in the $8,000 \mathrm{~K}$ to $10,000 \mathrm{~K}$ temperature interval, and surely the next application shall be in $\omega$ Cen stars. An alternative method would be to look for He-rich HB stars by analyzing their location on the $\log T$ vs. $\log g$ relation, and this project is ongoing.

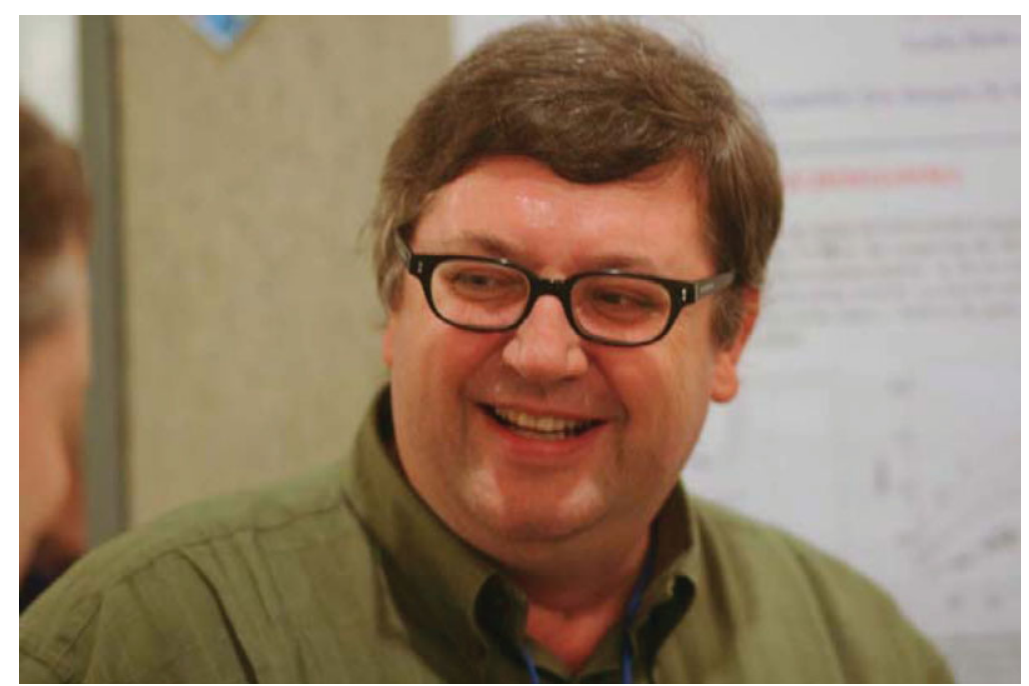

Giampaolo Piotto 